\title{
Are the responses generated during a psychophysical task independent of previously presented stimuli or do they depend on earlier stimulus presentations?
}

\author{
Sandra C M Quinn, Roger J Watt§ \\ School of Psychology, St Mary's College, University of St Andrews, Scotland, UK; \\ e-mail: sq4@st-andrews.ac.uk; §Department of Psychology, University of Stirling, Stirling FK9 4LA, \\ Scotland, UK
}

Received 19 September 2011, in revised form 15 February 2012

\begin{abstract}
Quinn and Watt (2006 Perception 35 267-280) showed that the optimal tempi for pieces of music (ie the appropriate speed for a melody) vary from melody to melody. The current study tested if such tempi responses depend on previous stimulus presentations. To do this, we ran a many-participants single-presentation version of the Quinn and Watt experiment. 616 visitors to the Glasgow Science Centre participated. We found that the results substantially matched those obtained earlier, and demonstrate that participants' responses are essentially independent of previously presented stimuli.
\end{abstract}

Keywords: psychophysics, previous stimulus presentation, optimal tempo

In almost all psychophysical experiments, each participant is given many trials to complete. Although this method is widely used in experimental research, it has been criticised because previous exposure to a stimulus can bias future responses (eg Warren 1970). The only response that is unaffected by previously presented stimuli is the response given on the first trial. In making only one response to a single stimulus presentation, any possibility of biasing future responses would be eliminated. If the pooled responses of many participants, each responding to just one stimulus, produced similar results to an experiment where a small number of participants responded to each stimulus, then it would demonstrate that responses were unaffected by previously presented stimuli. The current study uses this method to test if the optimal tempi for pieces of music are affected by previously presented stimuli or result from the percept of the melodies' stimulus characteristics. We ran a many-participants single-presentation version of an experiment previously conducted (Quinn and Watt 2006; see also Quinn et al, in press, 2012), in which listeners were required to judge if melodies were presented too fast or too slow.

616 adult visitors at the Glasgow Science Centre (GSC) were recruited. The study was conducted in a quiet room at the GSC. The same 22 melodies used in the Quinn and Watt (2006) study were selected for use in the current study (see table 1). Each melody was presented at 5 different tempi including 30, 49, 70, 90, and 110 beats per minute (bpm). Stimuli were presented to participants through headphones. Participants were required to make a response by pressing one of two keys on a computer keyboard. The stimuli were presented in a fully randomised order. Participants were told that they would be asked to listen to a single melody and make one of two responses on the keyboard. After hearing the melody, participants were asked to make a response by indicating whether they thought it sounded 'too fast' or 'too slow'. Even if they were unsure whether the melody sounded 'too fast' or 'too slow', they were instructed to make just one response. 616 participants were recruited, so giving 616 trials, or 4 trials at each of the 7 tempi $\times 22$ melodies. Although psychophysical studies typically use a far larger number of repeated trials, we opted for a reduced number in order to make the study manageable.

The data produced psychometric functions for each melody, giving the proportion of participants responding 'too fast' as a function of the tempo for each melody. 
Table 1. Melodies used in the current study.

\begin{tabular}{ll}
\hline Melody & Melody title \\
\hline 1 & Angus Cameron's complements to Alex Webster (Hunter 1979) \\
2 & Bonnie Glenfarg (Hunter 1979) \\
3 & Lament for the death of Rev. Archie Beaton (Hunter 1979) \\
4 & Lament of Flora MacDonald (Hunter 1979) \\
5 & MacPherson's Rant (Hunter 1979) \\
6 & Mairi Bhan Og Mary, young and fair (Hunter 1979) \\
7 & Mr Garden Troup's Farewell to France (Hunter 1979) \\
8 & Mrs Hamilton of Pentcaitland (Hunter 1979) \\
9 & Mrs Helen N Robertson (Hunter 1979) \\
10 & Roslin Castle (Hunter 1979) \\
11 & Sitting on the stern of a boat (Hunter 1979) \\
13 & The Duchess of Manchester's farewell to the highlands of Scotland (Hunter 1979) \\
14 & The fallen chief (Hunter 1979) \\
15 & The Marchoness of Huntly's favourite (Hunter 1979) \\
16 & The Marquee of Huntly's snuff mill (Hunter 1979) \\
17 & The Kilworth Hills (Martin 2002a) \\
18 & Miss Drummond of Perth (Martin 2002a) \\
19 & Loch Torridon (Martin 2002a) \\
21 & Queen Victoria's Diamond Jubilee (Martin 2002a) \\
22 & Stornoway Castle (Martin 2002b) \\
24 & Bob Steele (Martin 2002a) \\
25 & Lochanside (Martin 2002a) \\
& \\
\hline
\end{tabular}

Psychometric functions were fitted using the psignifit toolbox version 2.5.6 for Matlab (Hill 2005, see http://bootstrap-software.org/psignifit/) which implements the maximumlikelihood method described by Wichmann and Hill (2001a, 2001b). From these psychometric functions the point of subjective equality was calculated (ie $50 \%$ point on the curve), which we term the 'optimal tempo'.

If the psychometric functions obtained in the current study and the Quinn and Watt (2006, experiment 1) study (now referred to as QW2006) are similar, this would suggest that responses for previous stimuli are unlikely to affect responses for subsequent stimuli. Four psychometric functions are shown in figure 1. The PSE represents the point where the function crosses 0.5 and is shown on the graph as a solid line (see arrows). The optimal tempi for melody 1 (figure 1a) in the current study and the QW2006 study are similar (between 70 and $79 \mathrm{bpm}$ ). Melody 24 (figure 1b) has an optimal of $114 \mathrm{bpm}$ in both studies. It is clear that the fit of the function for melody 24 in the current study may actually be closer to the QW2006 study. This suggests that individual participants are capable of making 'too fast' and 'too slow' responses for a single stimulus that collectively yield an S-shaped psychometric function.

Establishing the similarity between the optimal in the current study and the QW2006 study would demonstrate that listeners can make responses that are independent of the responses generated by previously presented stimuli. Figure 1c shows that the relationship between the optimal in both studies is similar overall. The correlation between the optimal in the current and QW2006 study revealed a strong relationship $(r=0.61$, $p<0.001$ ), suggesting that the optimal in both studies were similar. Additionally, the optimal in the current and QW2006 study was not significantly different $\left(t_{21}=-0.96\right.$, $p>0.05)$. Therefore, the null hypothesis that the optimal in the current study and the QW2006 study are the same can be accepted. The present data also indicate that we can be $95 \%$ confident that the effect of using the two methods (single-shot and multi-shot tasks) changes the result by less than $11.8 \mathrm{bpm}$ or $13 \%$.

Taken together, these results suggest that judging the timing of each stimulus presentation in the QW2006 study is unlikely to be influenced by previously presented stimuli. The results suggest that listeners compare the physical tempi of a melody 


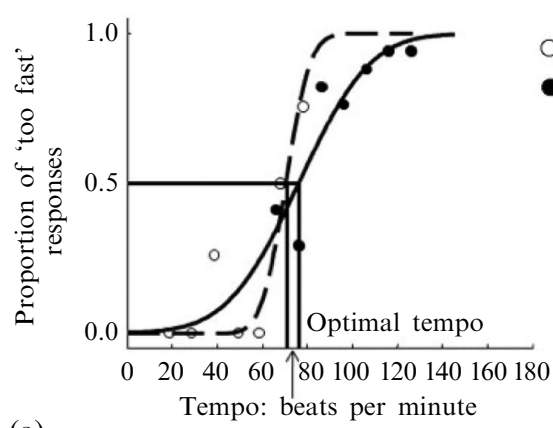

(a)

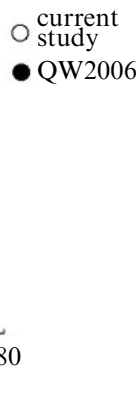

(b)
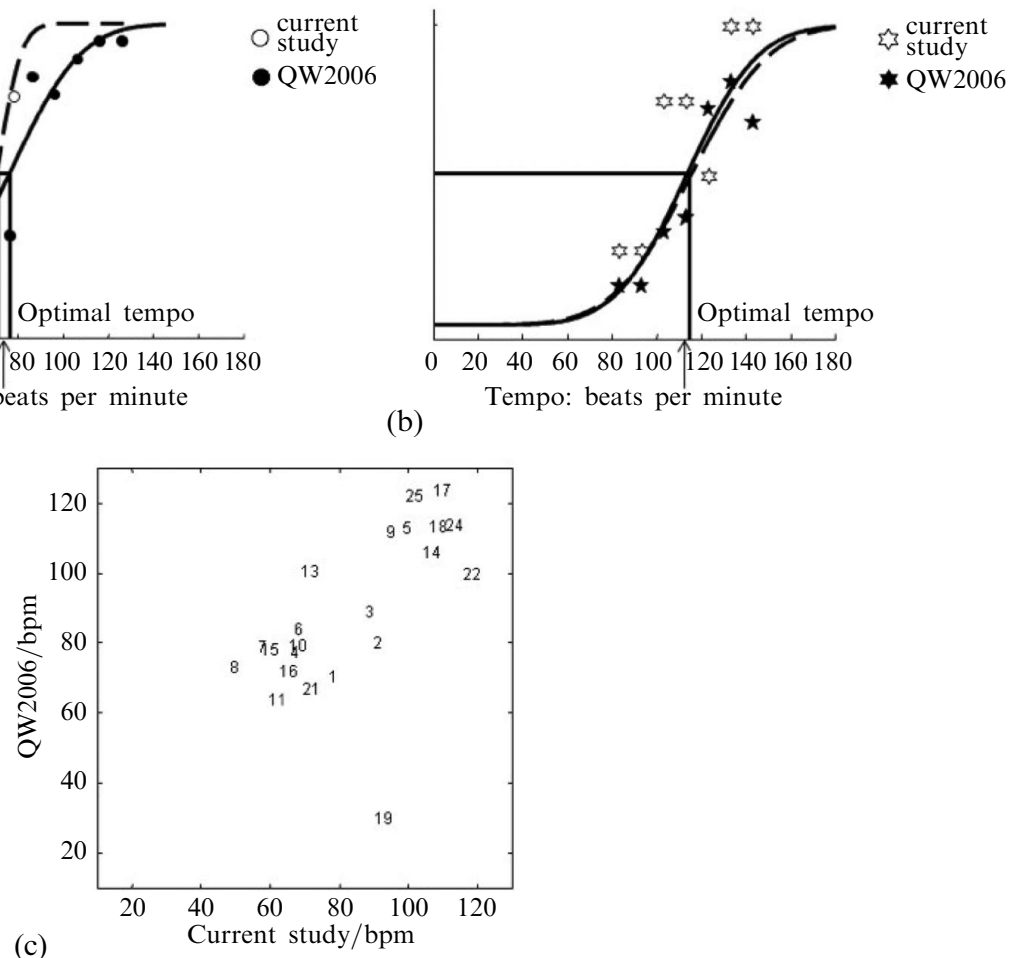

Figure 1. The proportion of 'too fast' responses as a function of tempo for melody 1 (figure 1a) and 24 (figure 1b) in the current study (dashed function) and the QW2006 study (solid function). The lower figure shows the optimal tempi in the current study and the QW2006 study.

with an internal optimal for that melody. In this way, listeners seem to make 'too fast' or 'too slow' responses based on the features of a melody and not a comparison between the tempi of previous stimuli with the current stimulus.

Acknowledgments. Thanks are given to the Glasgow Science Centre for their assistance in obtaining a large cohort of participants and providing space to complete the study. RJW was supported by a Leverhulme Research Fellowship during the course of this research.

\section{References}

Hill N J, 2005 "The Psignifit Toolbox" (Oxford: University of Oxford) http://bootstrap-software.org/ psignifit/ (last viewed 07/07/10)

Hunter J, 1979 The Fiddle Music of Scotland (Edinburgh: T and A Constable)

Martin C, 2002a The Fiddle Music of the Scottish Highlands; Ceol Na Fidhle 1 and 2 (Isle of Skye: Taigh Na Teud)

Martin C, 2002b The Fiddle Music of the Scottish Highlands; Ceol Na Fidhle 3 and 4 (Isle of Skye: Taigh $\mathrm{Na}$ Teud)

Quinn S, O'Haire O, Riby D M, in press, 2012 "How comparable are children and adults in perceiving an optimal tempo for music?" Journal of the Acoustical Society of America

Quinn S, Watt R J, 2006 "The perception of tempo in music” Perception 35 267-280

Warren R M, 1970 "Elimination of biases in loudness judgments for tones" Journal of the Acoustical Society of America $\mathbf{6 8} 1397-1403$

Wichmann F A, Hill N J, 2001a "The psychometric function: I. Fitting, sampling and goodness of fit" Perception \& Psychophysics $631293-1313$

Wichmann F A, Hill N J, 2001b "The psychometric function: II. Boostrap-based confidence intervals and sampling” Perception \& Psychophysics $631314-1329$ 


\section{PERCEPTION}

VOLUME 412012

www.perceptionweb.com

Conditions of use. This article may be downloaded from the Perception website for personal research by members of subscribing organisations. Authors are entitled to distribute their own article (in printed form or by e-mail) to up to 50 people. This PDF may not be placed on any website (or other online distribution system) without permission of the publisher. 